Article

\title{
Identification and Characterization of Cannabimovone, a Cannabinoid from Cannabis sativa, as a Novel PPAR $\gamma$ Agonist via a Combined Computational and Functional Study
}

\author{
Fabio Arturo Iannotti ${ }^{1,2}{ }^{\mathbb{D}}$, Fabrizia De Maio ${ }^{1,2}$, Elisabetta Panza ${ }^{3}$, Giovanni Appendino ${ }^{4}$, \\ Orazio Taglialatela-Scafati ${ }^{3}\left(\mathbb{D}\right.$, Luciano De Petrocellis ${ }^{1,2}{ }^{-}$, Pietro Amodeo ${ }^{1, *(1)}$ and \\ Rosa Maria Vitale ${ }^{1, *(D)}$ \\ 1 Institute of Biomolecular Chemistry (ICB), National Research Council (CNR), 80078 Pozzuoli (NA), Italy \\ 2 Endocannabinoid Research Group (ERG), Institute of Biomolecular Chemistry, National Research \\ Council (ICB-CNR), Via Campi Flegrei 34, 80078 Pozzuoli (NA), Italy \\ 3 Department of Pharmacy, University of Naples “Federico II", Via D. Montesano 49, I-80131 Napoli, Italy \\ 4 Dipartimento di Scienze del Farmaco, Università del Piemonte Orientale, Largo Donegani 2, \\ 28100 Novara, Italy \\ * Correspondence: pamodeo@icb.cnr.it (P.A.); rmvitale@icb.cnr.it (R.M.V.)
}

Received: 30 January 2020; Accepted: 1 March 2020; Published: 3 March 2020

\begin{abstract}
Phytocannabinoids (pCBs) are a large family of meroterpenoids isolated from the plant Cannabis sativa. $\Delta^{9}$-Tetrahydrocannabinol (THC) and cannabidiol (CBD) are the best investigated phytocannabinoids due to their relative abundance and interesting bioactivity profiles. In addition to various targets, THC and CBD are also well-known agonists of peroxisome proliferator-activated receptor gamma (PPAR $\gamma$ ), a nuclear receptor involved in energy homeostasis and lipid metabolism. In the search of new pCBs potentially acting as PPAR $\gamma$ agonists, we identified cannabimovone $(\mathrm{CBM})$, a structurally unique abeo-menthane $\mathrm{pCB}$, as a novel PPAR $\gamma$ modulator via a combined computational and experimental approach. The ability of CBM to act as dual PPAR $\gamma / \alpha$ agonist was also evaluated. Computational studies suggested a different binding mode toward the two isoforms, with the compound able to recapitulate the pattern of H-bonds of a canonical agonist only in the case of PPAR $\gamma$. Luciferase assays confirmed the computational results, showing a selective activation of PPAR $\gamma$ by CBM in the low micromolar range. CBM promoted the expression of PPAR $\gamma$ target genes regulating the adipocyte differentiation and prevented palmitate-induced insulin signaling impairment. Altogether, these results candidate CBM as a novel bioactive compound potentially useful for the treatment of insulin resistance-related disorders.
\end{abstract}

Keywords: phytocannabinoids; cannabimovone (CBM); peroxisome proliferator-activated receptor gamma (PPAR $\gamma$ ); molecular docking; molecular dynamics; insulin resistance

\section{Introduction}

Peroxisome proliferation-activated receptors (PPARs) are ligand-activated transcription factors involved in the regulation of energy homeostasis and metabolism [1]. The binding of agonists to the PPAR ligand-binding domain (LBD) induces conformational changes, leading to the release of a corepressor and the recruitment of coactivator peptides. The LBD is a 12-helix bundle domain with a central core forming the ligand binding pocket. The inactive conformation is characterized by high flexibility of the short helix H12, part of the coregulator interaction surface (AF-2), along with helices $\mathrm{H} 3$ and $\mathrm{H} 11$, and the $\mathrm{H} 3-\mathrm{H} 4$ loop [2]. Ligand binding stabilizes H12, triggering the 
switch toward the PPAR's active conformation. After binding to the agonist, PPARs heterodimerize with another nuclear receptor, the retinoid $X$ receptor (RXR), to modulate the transcription of its target genes [3]. Three different PPAR isoforms, namely $\alpha, \gamma$, and $\beta / \delta$, were characterized so far [4], with PPAR $\gamma$ playing a crucial role in regulating adipocyte differentiation, lipid metabolism, glucose homeostasis, and insulin resistance [5]. For these reasons, it represents a pharmacological target for the treatment of insulin resistance and dyslipidemia [6,7]. However, the therapeutic use of potent PPAR $\gamma$ agonists such as thiazolidinediones is restricted due to their severe side effects including weight gain, fluid retention, edema, and myocardial infarction [8,9]. Thus, a promising strategy consists of the development of less potent PPAR $\gamma$ modulators endowed with an acceptable safety profile. In this view, natural products represent an invaluable source of bioactive molecules due to their high chemical diversity. Among them, phytocannabinoids (pCBs) deserve special interest, since their major compounds, $\Delta^{9}$-tetrahydrocannabinol (THC) and cannabidiol (CBD), show a rich polypharmacology [10-13] including PPAR $\gamma$ agonism [14,15]. However, when considering the diversity of the chemical space of phytocannabinoids [16], their pharmacological potential as PPAR $\gamma$ is still substantially untapped. In this context, we focused our attention on cannabimovone (CBM), a pCB from fiber hemp (Cannabis sativa variety "Carmagnola") with a unique abeo-menthane terpenoid skeleton substantially different from that of THC and CBD [17], scouting its potency to act as a PPAR $\gamma$ agonist via molecular docking and molecular dynamics simulations, followed by validating the results with cellular assays. The PPAR $\alpha$ isoform was also evaluated to identify a possible dual profile of $\operatorname{PPAR} \alpha / \gamma$ modulation.

\section{Results}

\subsection{Molecular Docking and Molecular Dynamics}

The potential ability of cannabimovone (CBM, Figure 1) to bind and activate PPAR $\gamma$ and/or PPAR $\alpha$ was investigated via a computational approach based on a combination of molecular docking and molecular dynamics. For each receptor, the docking poses representative of the most populated clusters endowed with the most favorable binding energy were selected to undergo 50-ns molecular dynamics (MD) simulations to assess their stability, performing the MD analysis on the last $45 \mathrm{~ns}$. Unless differently stated, only the H-bonds with an occurrence $>10 \%$ during MD are reported.

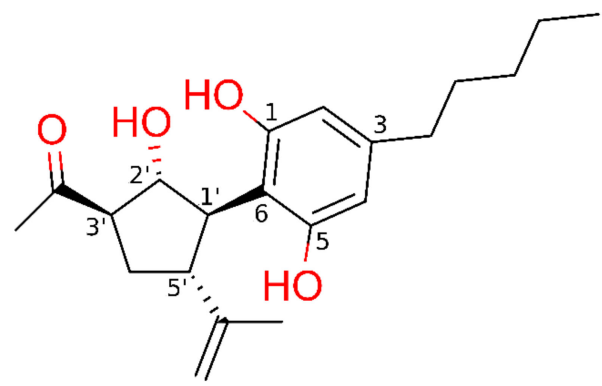

Figure 1. Chemical structure of cannabimovone (CBM) with functional groups colored in red.

\subsubsection{Theoretical Complexes of PPAR $\gamma$ with Cannabimovone (CBM)}

The analysis of docking results showed the occurrence of a single most populated cluster characterized by the best binding energy. The representative pose from the cluster was chosen to undergo the subsequent molecular dynamics (MD) simulation. After a slight initial rearrangement of the ligand alkyl chain, the complex remained stable through the last 45 ns (see Figure 2). The representative frame from MD, selected as described in Section 4, is reported in Figure 3. The polar groups of CBM engage stable H-bonds with Tyr473 (H12), a residue critical for PPAR $\gamma$ activation, along with His449 (H10-11) and Ser289 (H3). In detail, the CBM carbonyl group engages an H-bond with the hydroxyl group of Ser289 (H3) (occurrence of 66\%), the CBM hydroxyl group in 2' forms an 
H-bond network with both Tyr473 (H12) (occurrence of 75\%) and His449 (H10-11) (occurrence of 52\%) sidechains, and the CBM hydroxyl group in 1 forms H-bonds alternatively with the backbone carbonyl of Cys285 (H3) (occurrence of 49\%) or Ser289 (H3) sidechain (occurrence of 17\%). The isopropenyl group forms van der Waals interactions with both Phe282 (H3) and Phe363 (H7), while the pentyl chain interacts with Val339 in the $\beta$-sheet.

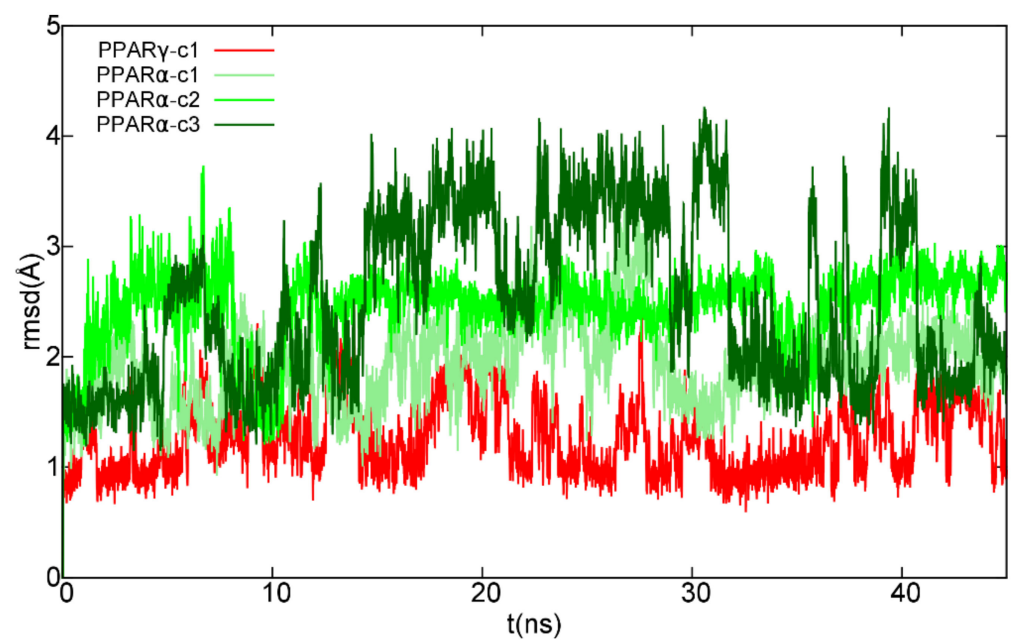

Figure 2. Root-mean-square deviation (RMSD) plot of CBM in complex with peroxisome proliferation-activated receptor (PPAR) $\gamma / \alpha$ over the last $45 \mathrm{~ns}$ of molecular dynamics (MD) trajectory after best fitting of protein backbones. The ligand RMSD plot was smoothed with a five-point window running average. Red color is used for CBM in complex with PPAR $\gamma$, while different shades of green are used for the ligand in the three simulated PPAR $\alpha$ complexes.

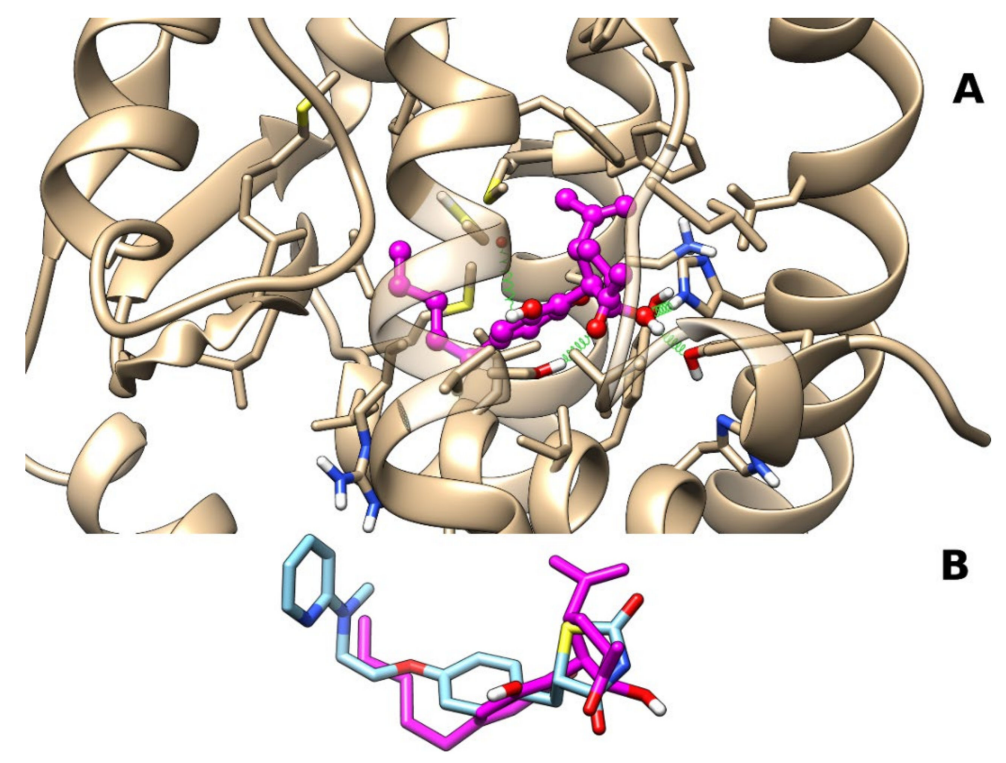

Figure 3. Representative frame from MD of PPAR $\gamma-C B M$ complex. Panel (A) A ball-and-stick representation is used for heavy atoms of the ligand, and a stick representation is used for protein sidechains within $5 \AA$ of the ligand. Protein carbon atoms are colored in tan according to the ribbon for the protein and in magenta for CBM. Hydrogen, nitrogen, oxygen, and sulfur atoms are painted white, blue, red, and yellow, respectively. Half-transparency is employed for the ribbon representation of protein regions overlying the ligand in the selected view. A "green spring" representation is adopted for H-bonds involving ligand atoms. Panel (B) Stick representation of CBM and rosiglitazone (Protein Data Bank (PDB) identifier (ID): 5ycp) after best fit of protein backbone. Carbon atoms of rosiglitazone are colored in light blue, while heteroatoms are colored according to panel (A). 


\subsubsection{Theoretical Complexes of PPAR $\alpha$ with Cannabimovone (CBM)}

The cluster analysis of docking results showed more dispersed binding poses in comparison with PPAR $\gamma$, with the three most populated clusters within $0.4 \mathrm{kcal} / \mathrm{mol}$ of binding energy. The representative frames from MD of each cluster complex are shown in Figure 3 and are hereinafter referred to as clust1, clust2, and clust3. Clust2 and clust3 show a very similar overall arrangement of the ligand in the LBD (see Figure 4B), but differ from each other in the orientation of the carbonyl group, whereas clust1 is shifted more toward helices H7/H10-11 (see Figure 4A,B). In all poses, the five-membered ring is tilted compared to the pose adopted in PPAR $\gamma$, possibly due to the difference in steric hindrance of Ile354 (H7) in place of PPAR $\gamma$ Phe363 and Tyr314(H5) in place of PPAR $\gamma$ His323 (H5), respectively. In both clust1 and clust2, the CBM carbonyl oxygen engages an H-bond with His440 (H10/11) with an occurrence of $71 \%$ and $48 \%$, respectively, but not with Tyr464 (H12) as in the case of the hydroxyl group in $2^{\prime}$ in PPAR $\gamma$. In clust3, CBM adopts a similar arrangement to clust 2 but it is shifted more toward the $\beta$-sheet, and the carbonyl group no longer forms an H-bond with His440 (H10/11), but instead forms one with the sidechain of Ser280 (H3) (occurrence of 25\%). In clust1, the CBM hydroxyl group in 1 forms H-bonds alternatively with the backbone carbonyl of Cys276 (H3) (occurrence of 48\%) and Ser280 (H3) sidechain (occurrence of 21\%), while the hydroxyl group in 2 engages an H-bond with the backbone carbonyl of Ile354 (H7) (occurrence of 35\%). Clust2 and clust3 share with clust 1 the formation of an H-bond between the hydroxyl group in 1 and the backbone carbonyl of Cys276 (H3) with an occurrence of 53\% and 33\%, respectively.

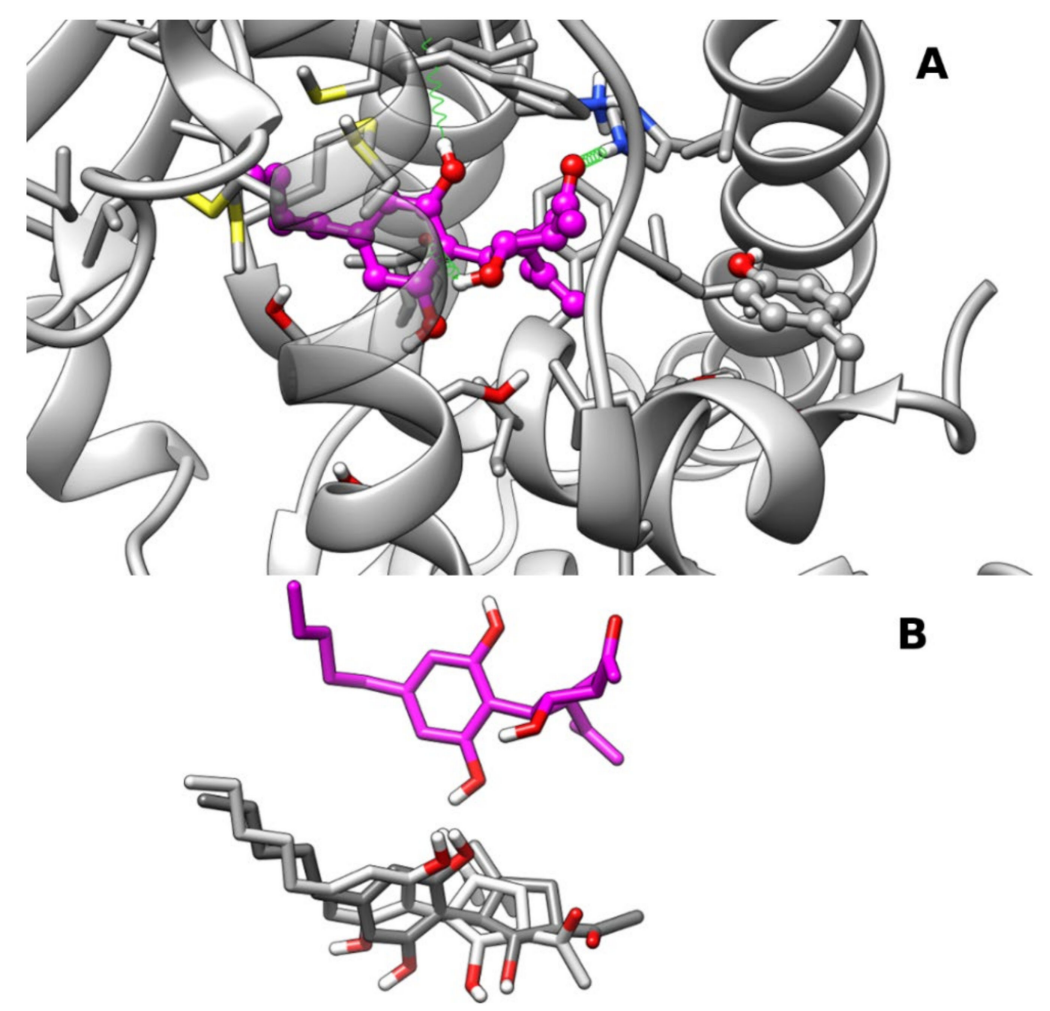

Figure 4. Representative frames from MD of PPAR $\alpha-C B M$ complexes: Panel (A) A ball-and-stick representation is used for the heavy atoms of the ligand, and a stick representation is used for the protein sidechain within $5 \AA$ of the ligand. Protein carbon atoms are colored in dark gray according to ribbon for protein and in magenta for CBM. Hydrogen, nitrogen, oxygen, and sulfur atom are painted white, blue, red, and yellow, respectively. Half-transparency is employed for the ribbon representation of protein regions overlying ligands in the selected view. A "green spring" representation is adopted for H-bonds involving ligand atoms. Panel (B) Stick representation of CBM poses in clust2 (light gray), clust3 (dark gray), and clust1 (magenta) after best fitting of protein backbone. CBM pose in clust1 was translated vertically for clarity. 


\subsection{Luciferase Assays}

To evaluate the potential cytotoxicity of CBM at the concentrations used for our functional assays, preliminary experiments of cell viability using the 3-(4,5-dimethylthiazol-2-yl)-2,5-diphenyltetrazolium bromide (MTT) assay were carried out on human embryonic kidney 293 (HEK293) cells. Therefore, HEK293 cells were exposed to increasing concentrations of $\mathrm{CBM}(1,5,10$, and $30 \mu \mathrm{M})$, and the results shown in Figure 5 demonstrate that CBM does not cause significant cytotoxic effects at any tested concentration. Subsequently, HEK293 cells were transiently transfected with the chimeric human PPAR $\alpha$-LBD-Gal4 or PPAR $\gamma$-LBD-Gal4 constructs concomitantly to a UAS enhancer (MH100). The day after transfection, cells were treated with CBM at 1, 5, 10, and $30 \mu \mathrm{M}$. Fenofibrate $(10 \mu \mathrm{M})$ and rosiglitazione $(0.01 \mu \mathrm{M})$ were used as positive controls for PPAR $\alpha$ and PPAR $\gamma$, respectively. As shown in Figure 6A,B, CBM promoted transcriptional activity in PPAR $\alpha$-transfected cells only at the highest assayed concentration $(30 \mu \mathrm{M})$. In contrast, the effect of CBM on PPAR $\gamma$ activity was much more prominent and concentration-dependent.

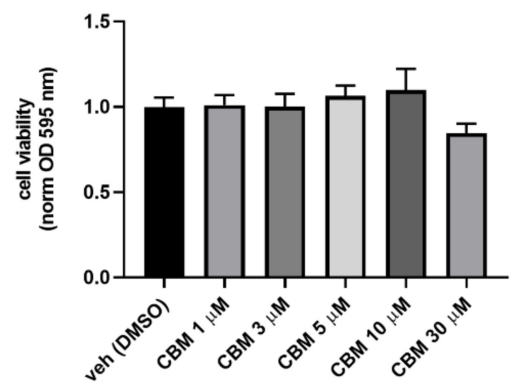

Figure 5. Cell viability measured in HEK293 cells treated for $24 \mathrm{~h}$ with crescent concentration of CBM. Bar graph shows cell viability measured using the 3-(4,5-dimethylthiazol-2-yl)-2,5-diphenyltetrazolium bromide (MTT) assay. Data are expressed as optical density (OD) at $595 \mathrm{~nm}$, normalized to control (vehicle). Each point is the mean \pm standard error of the mean (SEM) of four separate determinations performed in duplicate. Statistically significant differences were accepted when the $p$-value was at least $\leq 0.05$.

A

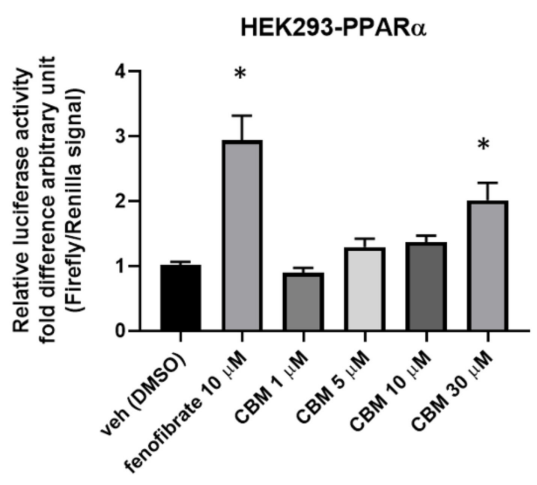

B

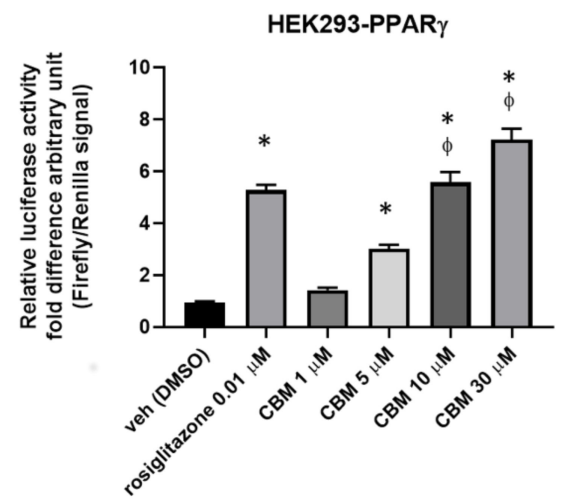

Figure 6. Luciferase assays performed in PPAR $\alpha$ - (A) or PPAR $\gamma$-transfected (B) HEK293 cells. Bar graphs showing the ratio between firefly and Renilla luciferase in response to crescent concentrations of cannabimovone (CBM; up to $30 \mu \mathrm{M})$. Fenofibrate and rosiglitazone were used as positive controls for $\operatorname{PPAR} \alpha$ and PPAR $\gamma$, respectively. The vehicle group was set to 1 ; thus, the relative luciferase activities obtained for each tested compound and concentration are presented as a fold induction with respect to the vehicle control. Each point is the mean \pm SEM of four separate determinations performed in duplicate. Statistically significant differences were accepted when the $p$-value was at least $\leq 0.05$. The asterisk $\left({ }^{*}\right)$ denotes a $p$-value $\leq 0.05$ vs. vehicle group; the symbol $\phi$ denotes $p \leq 0.05$ vs. vehicle group vs. CBM $5 \mu \mathrm{M}$. 


\subsection{CBM Upregulates Genes Involved in Adipocyte Differentiation and Energy Metabolism}

It is well known that, once activated by a ligand, PPAR $\gamma$ receptors bind to specific PPAR response elements (PPRE) to control the transcriptional activity of an array of genes, orchestrating a plethora of metabolic responses that impact the regulation of insulin sensitivity, as well as lipid and cholesterol metabolism. Furthermore, PPAR $\gamma$ activity is also important to trigger the differentiation and function of pre-adipocytes $[18,19]$. Therefore, to further explore the pharmacological activity of CBM on PPAR $\gamma$, we induced 3T3-L1 pre-adipocytes to differentiate for 10 days in the presence of rosiglitazone at $1 \mu \mathrm{M}$, and CBM at both 10 and $30 \mu \mathrm{M}$, to further explore the pharmacological activity of CBM on PPAR $\gamma$. After this time, the total RNA was isolated from each experimental condition and the expression of transcripts encoding for key PPAR $\gamma$-regulated gene markers of adipogenesis, including CCAAT/enhancer binding protein alpha $(\mathrm{C} / \mathrm{EBP} \alpha)$, fatty acid-binding protein 4 (FABP4), glucose transporter 4 (GLUT4), fatty acid synthase (FAS), and adiponectin [20,21], was measured by quantitative PCR (qPCR). The data revealed that, in 3T3-L1 cells induced to differentiate in the presence of rosiglitazone, as expected, the expression of all the mature adipocytes markers was robustly increased compared to the control group (Figure 7). In CBM-treated cells we observed that the increase in the expression of $\mathrm{cEBP} \alpha$, adiponectin, and FAS (only at $10 \mu \mathrm{M}$ ) was comparable to that observed with rosiglitazione (Figure 7). Less robust but still evident when compared to the control group was the expression of FABP4 and GLUT4. It is noteworthy that CBM, but not rosiglitazone, significantly increased the expression of PPAR $\gamma$, revealing that, most likely, this phytocannabinoid not only activates PPAR $\gamma$, but it is also able to trigger a positive feedback loop promoting gene expression.
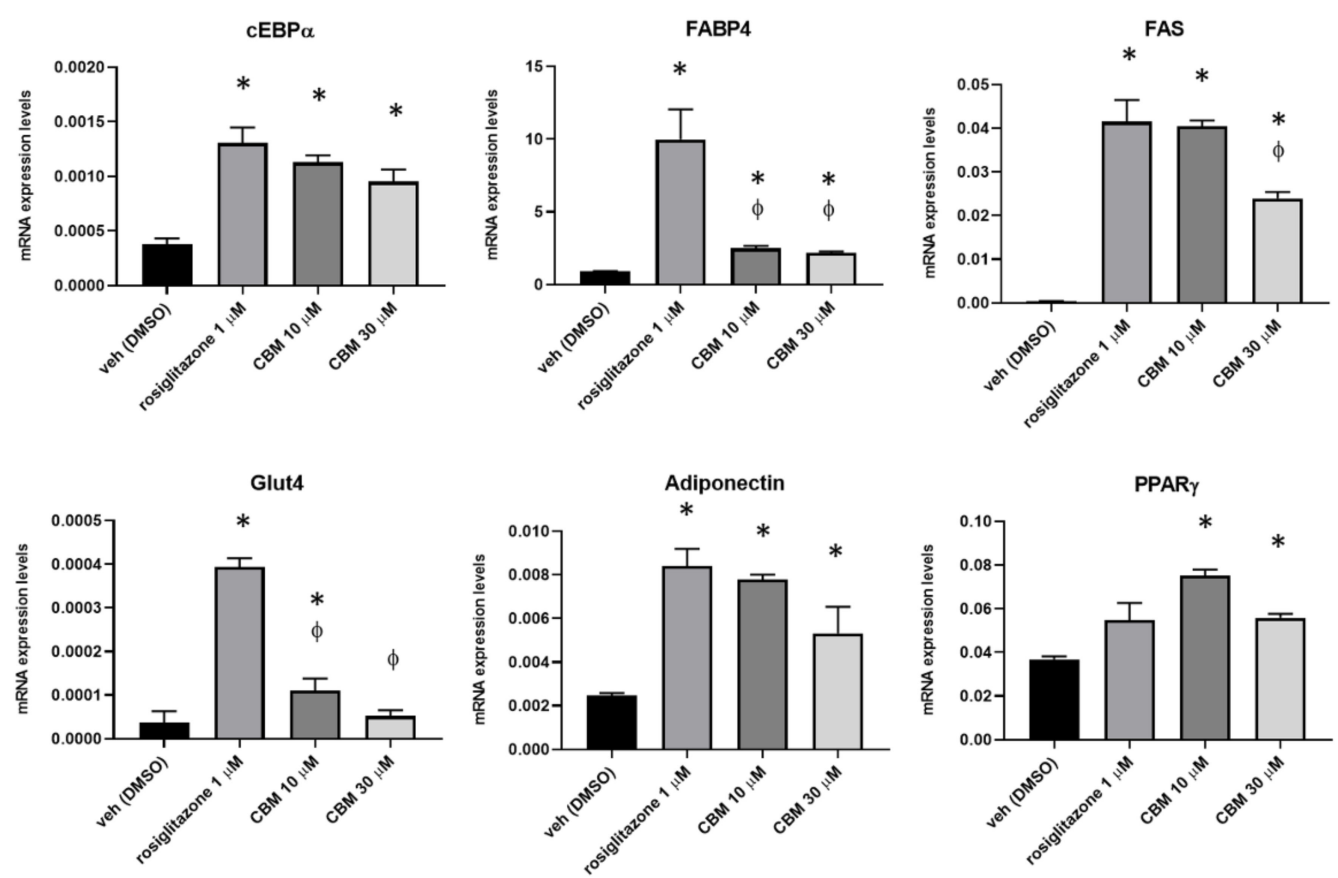

Figure 7. Effect of rosiglitazone and CBM in differentiating 3T3-L1 cells. Transcript levels of CCAAT/enhancer binding protein alpha $(\mathrm{C} / \mathrm{EBP} \alpha)$, fatty acid- binding protein 4 (FABP4), glucose transporter 4 (GLUT4), fatty acid synthase (FAS), adiponectin, and PPAR $\gamma$ in murine 3T3-L1 cells exposed to differentiation media (DM) in the presence of vehicle (dimethyl sulfoxide (DMSO)), rosiglitazone $1 \mu \mathrm{M}$, or CBM 10 and $30 \mu \mathrm{M}$. The quantification of transcripts was performed by qPCR. Data represent the mean \pm SEM of four independent determinations. Data are expressed as $2^{-\wedge} \Delta \mathrm{ct}$ relative to S16, as described in Section 4. Datasets were compared with one-way ANOVA followed by Tukey's test. Differences were considered statistically significant when $p \leq 0.05$. The asterisk * denotes a $p$-value $\leq 0.05$ vs. vehicle group; the symbol $\phi$ denotes $p \leq 0.05$ vs. vehicle group vs. CBM $5 \mu \mathrm{M}$. 


\subsection{CBM Improves Insulin Sensitivity in Differentiating 3T3-L1 Cells}

Finally, we explored whether, in differentiated 3T3-L1 cells, the insulin signaling impairment induced by palmitate could be prevented by CBM via PPAR $\gamma$ activation. In this view, 3T3-L1 cells were firstly induced to differentiate for 10 days. Subsequently, the cells were incubated in fresh differentiation media in the presence of rosiglitazone $0.01 \mu \mathrm{M}$ or CBM $10 \mu \mathrm{M}$. After $2 \mathrm{~h}$, sodium palmitate (NaP $350 \mu \mathrm{M}$ ) was added to each cell plate and incubated for a further $18 \mathrm{~h}$ [22]. As shown in Figure $8 \mathrm{~A}$, we found that, in differentiated 3T3-L1 cells, palmitate significantly reduced the phosphorylation of protein kinase B (Akt), physiologically required for insulin-mediated glucose uptake [23]. However, in the presence of rosiglitazone and CBM, the phosphorylation of Akt was fully recovered. Along with this, we measured the glucose uptake under the same experimental conditions. As shown in Figure 8B, palmitate significantly inhibited $(100 \mathrm{nM})$ insulin-evoked uptake of glucose. This latter effect was fully prevented by rosiglitazone and also significantly prevented by CBM $10 \mu \mathrm{M}$. CBM $30 \mu \mathrm{M}$ produced no further effects (data not shown).

A

B

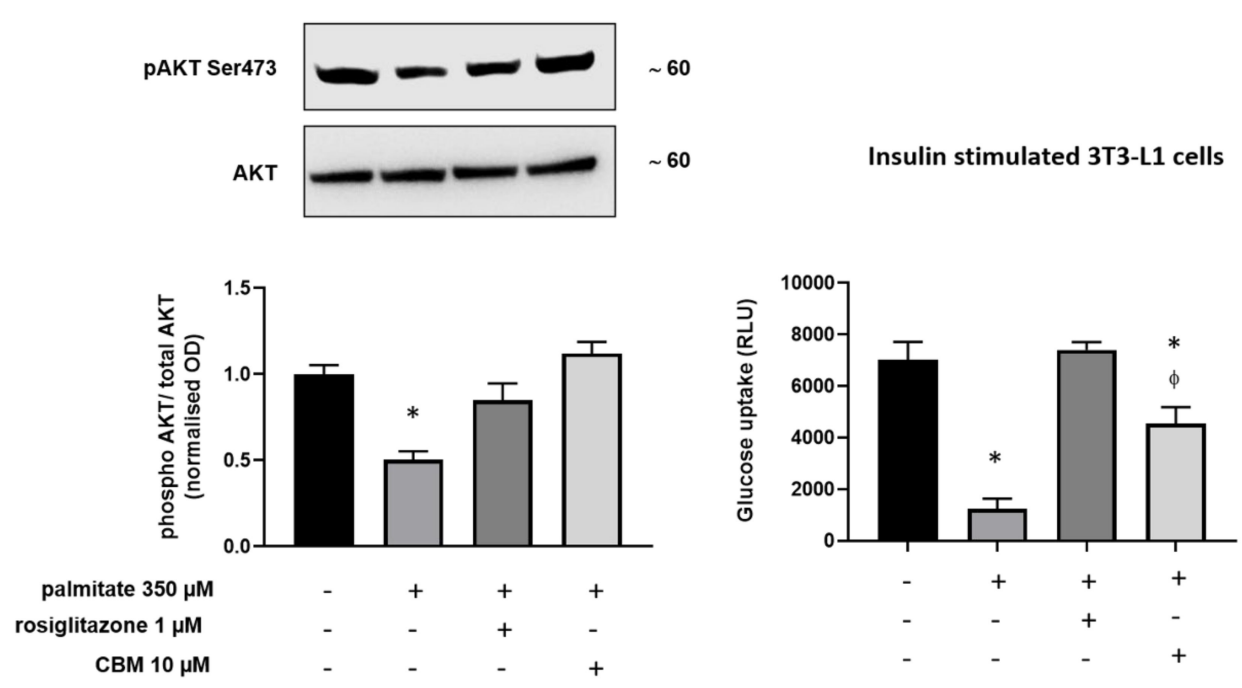

Figure 8. Measurement of protein kinase B (Akt) phosphorylation in differentiated 3T3-L1 cells exposed to sodium palmitate (NaP) in the presence or absence of rosiglitazone and CBM. (A) Upper representative blot showing the band intensity of phospho-(Ser473) and total Akt in differentiated 3T3-L1 cells treated with sodium palmitate $350 \mu \mathrm{M}$ for $18 \mathrm{~h}$. Lower bar graph showing the quantification of phospho-Akt levels normalized to Akt (total). (B) Levels of glucose uptake expressed as relative luminescence units (RLU) in insulin-stimulated 3T3-L1 cells. Data represent the mean \pm SEM of three separate determinations. Datasets were compared by one-way ANOVA followed by Tukey's test. Differences were considered statistically significant when $p \leq 0.05$. The asterisk $\left(^{*}\right)$ denotes a $p$-value $\leq$ 0.05 vs. vehicle control group (left) or stimulated 3T3-L1 cells with insulin $100 \mathrm{nM}$ (right); the symbol $\phi$ denotes a $p$-value $\leq 0.05$ vs. rosiglitazone.

\section{Discussion}

Phytocannabinoids (pCBs) are a class of secondary metabolites isolated from C. sativa, counting more than 100 compounds with a still largely unexplored pharmacological potential [16]. THC and $\mathrm{CBD}$, the most representative pCBs, have interesting therapeutic applications due to their complex pharmacological profile, and they are active on the PPAR $\gamma$ receptor, while a few other neutral and acidic pCBs were characterized as dual PPAR $\alpha / \gamma$ agonists [15]. Due to its role in adipocyte differentiation, lipid metabolism, glucose homeostasis, insulin sensitivity, and, more recently, in inflammatory and immune responses, PPAR $\gamma$ represents an attractive pharmacological target to address metabolic disorders. As part of our discovery program on nuclear receptor ligands from marine [24,25] and terrestrial sources [15], we focused on cannabimovone (CBM), a polar pCB characterized by a unique 
abeo-menthane terpenyl moiety isolated in 2010 from a non-psychotropic variety of C. sativa (Italian cultivar Carmagnola) [17]. The chemical structure of cannabimovone, including stereochemical details, was recently confirmed by total synthesis, which can also be considered as an alternative to the exploitation of the natural source [26]. The scarcity of pharmacological data available on CBM prompted us to investigate in silico the suitability of this scaffold to act as a potential selective or dual PPAR $\alpha / \gamma$ agonist. From docking and molecular dynamics studies, a difference in the binding modes between the two isoforms emerged, suggesting a stronger activity toward PPAR $\gamma$ in comparison to PPAR $\alpha$. In fact, while, in the PPAR $\gamma$ complex, CBM is able to directly interact through H-bond with Tyr473 (H12), the key residue located on the AF-2 domain and responsible for the activation of the receptor by full agonists, in the PPAR $\alpha$ complex, a different arrangement of the ligand was observed, with the lack of the aforementioned interaction. Moreover, in all the simulated PPAR $\alpha$ complexes, CBM is characterized by a higher mobility than in the PPAR $\gamma$ complex, as shown in the root-mean-square deviation (RMSD) plot (Figure 2). To validate the computational results, luciferase assays on both PPAR $\gamma$ and PPAR $\alpha$ were carried out, confirming the higher potency of CBM toward PPAR $\gamma$ in comparison to PPAR $\alpha$ (Figure 6) with a dose-dependent activation of Gal4-PPAR $\gamma$ in the former case. However, when compared to rosiglitazone, CBM is a weaker PPAR $\gamma$ agonist, and this can be mainly ascribed to its reduced size, which prevents the full occupancy of the active site. In particular, the alkyl chain has only a limited interaction with the $\beta$-sheet region of the LDB (see Figure 3), usually deeply involved in hydrophobic interactions with potent PPAR $\gamma$ agonists. However, in principle, a lower potency should be associated with a better safety profile. In this view, the cell viability assay (MTT) assay showed no-toxicity of CBM up to $30 \mu \mathrm{M}$, while, for other phytocannabinoids, we found a toxic effect even at $10 \mu \mathrm{M}$ [15]. Then, we further evaluated the effect of CBM on PPAR $\gamma$ downstream genes involved in adipocyte differentiation, such as $\operatorname{PPAR} \gamma, \mathrm{C} / \mathrm{EBP} \alpha$, and FAPB4. Our results indicate that CBM promotes adipocyte differentiation in 3T3-L1 by increasing PPAR $\gamma$ transcriptional activity at the aforementioned genes. Adipose tissue insulin resistance is characterized by a deficiency in GLUT4 [27], and rosiglitazone, a well-known insulin-sensitizer, acts at least in part by enhancing GLUT4 expression [28]. In the present study, we found that also CBM upregulates GLUT4 and adiponectin expression, thus promoting insulin-stimulated glucose transport in 3T3-L1 adipocytes. Moreover, since numerous studies suggest that the phosphorylation-mediated activation of Akt, which in turn positively regulates downstream targets including Glut4, glycogen synthase kinase 3 (GSK3), forkhead box O1 (Foxo1), hormone sensitive lipase (HSL), and mammalian target of rapamycin (mTOR), is one of the most important intracellular pathways promoting insulin sensitivity [29-31], we evaluated the effect of CBM on the insulin signaling impairment mediated by palmitate. In our study, CBM fully restored the reduced levels of Akt phosphorylation induced by palmitate, as well as increased the level of insulin-mediated glucose uptake (Figure 8A,B). In conclusion, our combined computational and experimental approach led to the identification and a structural and functional characterization of a novel phytocannabinoid as a PPAR $\gamma$ agonist able to in vitro stimulate insulin signaling, paving the way for further in vivo studies to assess the suitability of CBM as anti-diabetic and insulin-sensitizing drug, thus opening new therapeutic alternatives for those patients still not receiving an effective and safe long-term treatment.

\section{Methods}

\subsection{Purification of Cannabimovone}

CBM used in tests was obtained from the polar fraction of the acetone extract of a non-psychotropic variety of C. sativa. Isolation, purification, and structural elucidation of CBM were described elsewhere [17]. 


\subsection{Molecular Docking and Molecular Dynamics}

Starting ligand geometry was built with Ghemical 2.99.2 [32] followed by initial energy minimization (EM) at the molecular mechanics level, using Tripos 5.2 force field parametrization [33], and then at the AM1 semi-empirical level. The molecule was then fully optimized using the GAMESS program [34] at the Hartree-Fock level with the STO-3G basis set and subjected to HF/6-31G*/STO-3G single-point calculations to derive the partial atomic charges using the RESP procedure [35]. Docking studies were performed with AutoDock 4.2 [36], by using PPAR $\alpha$ and PPAR $\gamma$ crystallographic structures (Protein Data Bank (PDB): 2P54 and 2F4B, respectively). Both proteins and ligands were processed with AutoDock Tools (ADT) package version 1.5.6rc1 [36] to merge non-polar hydrogens and calculate Gasteiger charges. Grids for docking evaluation with a spacing of $0.375 \AA$ and $60 \times 60 \times 60$ points, centered on the ligand binding site, were generated using the program AutoGrid 4.2 included in Autodock 4.2 distribution. The Lamarckian genetic algorithm (LGA) was adopted to perform 100 docking runs with the following parameters: 100 individuals in a population with a maximum of 15 million energy evaluations and a maximum of 37,000 generations, followed by 300 iterations of Solis and Wets local search. The complexes, selected on the basis of binding energy and cluster population, were completed by addition of all hydrogen atoms, and they underwent energy minimization (EM) and then molecular dynamics (MD) simulations with Amber16 pmemd.cuda module [37], using the ff14SB version of the AMBER force field for the protein and gaff parameters [38] for the ligand.

To perform MD simulations in solvent, the complexes were confined in TIP3P water periodic truncated octahedron boxes exhibiting a minimum distance between solute atoms and box surfaces of $10 \AA$, using the tleap module of the AmberTools16 package. The systems were then neutralized by addition of counterions $\left(\mathrm{Na}^{+}\right)$and subjected to 1000 steps of EM with solute atoms harmonically restrained to their starting positions $\left(\mathrm{K}_{\mathrm{r}}=10 \mathrm{kcal} \cdot \mathrm{mol}^{-1} \cdot \AA^{-1}\right)$. Then, a 500 -ps restrained MD simulation $\left(\mathrm{K}_{\mathrm{r}}=5 \mathrm{kcal} \cdot \mathrm{mol}^{-1} \cdot \AA^{-1}\right)$ at constant pressure was run on each solvated complex, gradually heating the system to $300 \mathrm{~K}$, followed by a 500-ps restrained MD simulation $\left(\mathrm{K}_{\mathrm{r}}=5 \mathrm{kcal} \cdot \mathrm{mol}^{-1} \cdot \AA^{-1}\right)$ at constant temperature $(300 \mathrm{~K})$ and pressure $(1 \mathrm{~atm})$ to adjust system density. Production MD simulations were carried out at constant temperature $(300 \mathrm{~K})$ and pressure $(1 \mathrm{~atm})$ for $50 \mathrm{~ns}$, with a time step of $2 \mathrm{fs}$. Bonds involving hydrogens were constrained using the SHAKE algorithm [39]. The cpptraj module of AmberTools16 and program UCSF Chimera 1.10.1 [40] were used to perform MD analysis and to draw the figures, respectively. Cluster analysis was carried out with the cpptraj module using the dbscan clustering algorithm using the following parameters: minpoints 25, epsilon 0.9, sieve 10 . The representative frames were taken from the most populated clusters of each MD simulation.

\subsection{Cell Culture, Transfection, and Luciferase Assay}

Human embryonic kidney 293 cells (HEK293) were propagated in in a growth medium (GM) composed of Dulbecco's modified Eagle's medium (DMEM) supplemented with 10\% fetal bovine serum and $1 \%$ penicillin/streptomycin under standard conditions. After plating, the cells were transfected on the next day with the following plasmids: (a) pM1-hPPAR $\alpha$-Gal4 or pM1-hPPAR $\gamma$-Gal4, (b) TK-MH100 × 4-Luc containing the UAS enhancer elements, and (c) Renilla luciferase (pRL, Promega, Cat. E2231) using lipofectamine 2000 (cat. n. 11668027; Life Technologies; Milan, Italy) following the manufacturer's instructions. The next day, the growth medium was replaced with fresh medium containing compounds of interest. Dimethyl sulfoxide (DMSO) was used as a vehicle. On the third day, the cells were harvested and processed for analyzing the luciferase activity using a GloMax Luminometer instrument (Promega) and the Dual-Luciferase Reporter Assay kit (cat. n. E1910 Promega, IT) [15].

\subsection{MTT Assay}

HEK293 cells were seeded at $2 \times 10^{3}$ cells $/ \mathrm{cm}^{2}$ density in 24 -well plastic plates. One day after plating, CBM (up to $30 \mu \mathrm{M}$ ) was added to the culture medium for $24 \mathrm{~h}$. Cell viability was evaluated with 
the 3-(4,5-dimethylthiazol-2-yl)-2,5-diphenyltetrazolium bromide (MTT; $0.5 \mathrm{mg} / \mathrm{mL}$; Sigma-Aldrich) reduction assay, and formazan salt formation upon MTT reduction by the mitochondria of living cells was detected spectrophotometrically at $595 \mathrm{~nm}$ according to published procedures [41].

\subsection{RNA Purification and Quantitative Real-Time PCR ( $q P C R$ )}

Total RNA was isolated from cells using Pure Link ${ }^{\circledR}$ RNA Mini Kit (Cat. N.: 12183018A; Thermo Fisher Scientific, Milan, Italy) following the manufacturer's instruction, and then quantified by spectrophotometric analysis. The purified mRNA was reverse-transcribed using the iScript reverse transcriptase enzyme (Cat. N.: 1708840; Biorad, Milan, Italy). Quantitative real-time PCR was carried out in a CFX384 real-time PCR detection system (Bio-Rad, Milan, Italy) with specific primers (Table 1) using ssoAdvance Universal SYBR Green Supermix (Cat. N.: 1,725,270 Bio-Rad, Milan, Italy). Samples were amplified simultaneously in quadruplicate in a one-assay run with a non-template control blank for each primer pair to control for contamination or primer dimer formation, and the ct (cycle threshold) value for each experimental group was determined. Housekeeping genes (the ribosomal protein S16) were used as an internal control to normalize the ct values using the $2^{-\Delta c t}$ formula [41].

Table 1. Primers used in this study.

\begin{tabular}{|c|c|c|}
\hline Gene & Forward Sequence $\left(5^{\prime}-3^{\prime}\right)$ & Reverse Sequence $\left(5^{\prime}-3^{\prime}\right)$ \\
\hline PPAR $\gamma$ & GTCGGTTTCAGAAGTGCCTTG & GCTTTGGTCAGCGGGAAG \\
\hline$F A B P 4$ & TGTGATGCCTTTGTGGCAACCTG & TATGATGCTCTTCACCTTCCTGTCG \\
\hline$C / E B P \alpha$ & CAAGAACAGCAACGAGTACCG & GTCACTGGTCAACTCCAGCAC \\
\hline S16 & CTGGAGCCTGTTTTGCTTCTG & TGAGATGGACTGTCGGATGG \\
\hline GLUT4 & GCTCTGACGATGGGGAAC & CCAACACGGCCAAGACATTG \\
\hline FAS & GGAGGTGGTGATAGCCGGTAT & TGGGTAATCCATAGAGCCCAG \\
\hline Adiponectin & TGACGACACCAAAAGGGCTC & GAGTGCCATCTCTGCCATCA \\
\hline
\end{tabular}

\subsection{Western Blotting Analysis}

The 3T3-L1 cells were subjected to western blot analysis following the procedure previously described [41]. Briefly, following the treatment with palmitate, rosiglitazone, or CBM, differentiated 3T3-L1 cells were homogenized in lysis buffer composed of $1 \times$ Tris-HCl/NaCl/EDTA (TNE) buffer, $1 \%$ $(v / v)$ Triton X-100, protease (cat. n. P8340, Sigma-Aldrich, MI Italy), and phosphatase (cat. n. P5726, Sigma-Aldrich, MI Italy) inhibitor cocktails at $\mathrm{pH}$ 7.4. Lysates were kept in orbital shaker incubator at $220 \mathrm{rpm}$ at $4{ }^{\circ} \mathrm{C}$ for $30 \mathrm{~min}$ and then centrifuged for $15 \mathrm{~min}$ at $13,000 \times \mathrm{g}$ at $4{ }^{\circ} \mathrm{C}$. The supernatants were transferred to tubes and quantified by DC Protein Assay (Bio-Rad, Milan, Italy). Subsequently, the samples $\left(60 \mu \mathrm{g}\right.$ of total protein) were heated at $70{ }^{\circ} \mathrm{C}$ for $10 \mathrm{~min}$ in NuPAGE LDS Sample Buffer (cat. n. NP0007, Life Technology, Milan, Italy) plus Sample Reducing Agent (cat. n. NP0004, Life Technology MI Italy) and loaded onto 4\%-12\% Bis-Tris Protein Gels (cat. n. NP0336PK2, Life Technology, Milan, Italy) and then transferred to a polyvinylidene fluoride (PVDF) membrane. The primary antibodies used were (a) Akt (9272, Cell Signaling, USA; diluted 1:1000); (b) phospho-Akt (Ser473) XP (4060, Cell Signaling, USA; diluted 1:2000). Reactive bands were detected by enhanced chemiluminescence (ECL-plus; Bio-Rad, Segrate, Italy). The intensity of bands was analyzed on a ChemiDoc station with Quantity-one software (Biorad, Segrate, Italy).

\subsection{Glucose Uptake Assay}

The glucose uptake in 3T3-L1 was measured using the commercially available kit Glucose Uptake-Glo ${ }^{\mathrm{TM}}$ Assay (cat. J1341) purchased from Promega (Milan, Italy) following the manufacturer's indications. Briefly, 3T3-L1 were differentiated in 24-well culture plates following published procedures [23]. After $16 \mathrm{~h}$ of incubation with palmitate (plus Rosiglitazone or CBM), the cells were washed three times with PBS, and then stimulated with $100 \mathrm{nM}$ insulin at $37^{\circ} \mathrm{C}$ for $20 \mathrm{~min}$ [30]. The bioluminescent signal was acquired on a Glomax luminometer (Promega, Italy). 


\subsection{Chemical Reagents}

Dimethyl sulfoxide (cat. n. W387520), sodium palmitate (cat. n. P9767), fenofibrate (cat. n. F6020), and insulin (cat. n. I6634) were from Sigma Aldrich (Milan, Italy); rosiglitazone (cat. n. 5325) was from Tocris Bioscience (Bristol, UK).

\subsection{Statistical Analysis}

Data are expressed as means \pm S.E.M. of the given number of experiments $(n)$. Datasets were compared by use of matched Student's $t$-tests or, if necessary, with one-way analysis of variance, followed by Tukey's test. Statistically significant differences were accepted at $p \leq 0.05$.

Author Contributions: Conceptualization, F.A.I. and R.M.V.; Methodology, F.A.I., E.P., R.M.V. and P.A.; Validation, F.A.I., F.D.M., R.M.V. and P.A.; Formal Analysis, F.A.I., F.D.M., E.P., R.M.V.; Investigation, F.A.I., R.M.V., P.A.; Writing-Original Draft Preparation, F.A.I., R.M.V. and P.A.; Writing-Review \& Editing, O.T.-S., G.A., L.D.P., R.M.V. and P.A.; Visualization, F.A.I., R.M.V.; Funding Acquisition, O.T.-S., G.A., L.D.P., R.M.V. All authors have read and agreed to the published version of the manuscript.

Funding: This research was funded by MIUR, research grant PRIN2017, Project WN73PL (Bioactivity-directed exploration of the phytocannabinoid chemical space).

Acknowledgments: We are very grateful to Karsten Kristiansen, from the University of Copenhagen for the kind gift of pM1-hPPAR $\alpha$-Gal4 and to Michael Privalsky from the University of California for pM1-hPPAR $\gamma-G a l 4$ plasmids. Finally, we are deeply grateful to Marco Allarà for the help with the cell culture and Salvatore Donadio for the technical support.

Conflicts of Interest: The authors declare no conflict of interest.

\section{References}

1. Berger, J.; Moller, D.E. The Mechanisms of Action of PPARs. Annu. Rev. Med. 2002, 53, 409-435. [CrossRef] [PubMed]

2. Moore, J.T.; Collins, J.L.; Pearce, K.H. The Nuclear Receptor Superfamily and Drug Discovery. ChemMedChem 2006, 1, 504-523. [CrossRef] [PubMed]

3. Gearing, K.L.; Göttlicher, M.; Teboul, M.; Widmark, E.; Gustafsson, J.A. Interaction of the peroxisome-proliferator-activated receptor and retinoid X receptor. Proc. Natl. Acad. Sci. USA 1993, 90, 1440-1444. [CrossRef] [PubMed]

4. Braissant, O.; Foufelle, F.; Scotto, C.; Dauça, M.; Wahli, W. Differential expression of peroxisome proliferator-activated receptors (PPARs): Tissue distribution of PPAR-alpha, -beta, and -gamma in the adult rat. Endocrinology 1996, 137, 354-366. [CrossRef]

5. Willson, T.M.; Lambert, M.H.; Kliewer, S.A. Peroxisome Proliferator-Activated Receptor $\gamma$ and Metabolic Disease. Annu. Rev. Biochem. 2001, 70, 341-367. [CrossRef]

6. Janani, C.; Ranjitha Kumari, B.D. PPAR gamma gene-A review. Diabetes Metab. Syndr. Clin. Res. Rev. 2015, 9, 46-50. [CrossRef]

7. Staels, B.; Fruchart, J.-C. Therapeutic Roles of Peroxisome Proliferator-Activated Receptor Agonists. Diabetes 2005, 54, 2460-2470. [CrossRef]

8. Nissen, S.E.; Wolski, K. Effect of Rosiglitazone on the Risk of Myocardial Infarction and Death from Cardiovascular Causes. N. Engl. J. Med. 2007, 356, 2457-2471. [CrossRef]

9. Horita, S.; Nakamura, M.; Satoh, N.; Suzuki, M.; Seki, G. Thiazolidinediones and Edema: Recent Advances in the Pathogenesis of Thiazolidinediones-Induced Renal Sodium Retention. PPAR Res. 2015, 2015, 1-7. [CrossRef]

10. Devinsky, O.; Cilio, M.R.; Cross, H.; Fernandez-Ruiz, J.; French, J.; Hill, C.; Katz, R.; Di Marzo, V.; Jutras-Aswad, D.; Notcutt, W.G.; et al. Cannabidiol: Pharmacology and potential therapeutic role in epilepsy and other neuropsychiatric disorders. Epilepsia 2014, 55, 791-802. [CrossRef]

11. Devinsky, O.; Whalley, B.J.; Di Marzo, V. Cannabinoids in the Treatment of Neurological Disorders. Neurotherapeutics 2015, 12, 689-691. [CrossRef] [PubMed] 
12. Boggs, D.L.; Nguyen, J.D.; Morgenson, D.; Taffe, M.A.; Ranganathan, M. Clinical and Preclinical Evidence for Functional Interactions of Cannabidiol and $\Delta$ 9-Tetrahydrocannabinol. Neuropsychopharmacology 2018, 43, 142-154. [CrossRef] [PubMed]

13. Ibeas Bih, C.; Chen, T.; Nunn, A.V.W.; Bazelot, M.; Dallas, M.; Whalley, B.J. Molecular Targets of Cannabidiol in Neurological Disorders. Neurotherapeutics 2015, 12, 699-730. [CrossRef] [PubMed]

14. O'Sullivan, S.E. An update on PPAR activation by cannabinoids. Br. J. Pharmacol. 2016, 173, 1899-1910. [CrossRef]

15. D'Aniello, E.; Fellous, T.; Iannotti, F.A.; Gentile, A.; Allarà, M.; Balestrieri, F.; Gray, R.; Amodeo, P.; Vitale, R.M.; Di Marzo, V. Identification and characterization of phytocannabinoids as novel dual PPAR $\alpha / \gamma$ agonists by a computational and in vitro experimental approach. Biochim. Biophys. Acta Gen. Subj. 2019, 1863, 586-597. [CrossRef]

16. Hanuš, L.O.; Meyer, S.M.; Muñoz, E.; Taglialatela-Scafati, O.; Appendino, G. Phytocannabinoids: A unified critical inventory. Nat. Prod. Rep. 2016, 33, 1357-1392. [CrossRef]

17. Taglialatela-Scafati, O.; Pagani, A.; Scala, F.; De Petrocellis, L.; Di Marzo, V.; Grassi, G.; Appendino, G. Cannabimovone, a Cannabinoid with a Rearranged Terpenoid Skeleton from Hemp. Eur. J. Org. Chem. 2010, 2010, 2067-2072. [CrossRef]

18. Azhar, S. Peroxisome proliferator-activated receptors, metabolic syndrome and cardiovascular disease. Future Cardiol. 2010, 6, 657-691. [CrossRef]

19. Ciudin, A.; Hernandez, C.; Simo, R. Update on Cardiovascular Safety of PPARgamma Agonists and Relevance to Medicinal Chemistry and Clinical Pharmacology. Curr. Top. Med. Chem. 2012, 12, 585-604. [CrossRef]

20. Moseti, D.; Regassa, A.; Kim, W.-K. Molecular Regulation of Adipogenesis and Potential Anti-Adipogenic Bioactive Molecules. Int. J. Mol. Sci. 2016, 17, 124. [CrossRef]

21. Rosen, E.D. C/EBPalpha induces adipogenesis through PPARgamma: A unified pathway. Genes Dev. 2002, 16, 22-26. [CrossRef] [PubMed]

22. Chen, X.; Zhao, X.; Lan, F.; Zhou, T.; Cai, H.; Sun, H.; Kong, W.; Kong, W. Hydrogen Sulphide Treatment Increases Insulin Sensitivity and Improves Oxidant Metabolism through the CaMKKbeta-AMPK Pathway in PA-Induced IR C2C12 Cells. Sci. Rep. 2017, 7, 13248. [CrossRef] [PubMed]

23. Fellous, T.; De Maio, F.; Kalkan, H.; Carannante, B.; Boccella, S.; Petrosino, S.; Maione, S.; Di Marzo, V.; Iannotti, F.A. Phytocannabinoids promote viability and functional adipogenesis of bone marrow-derived mesenchymal stem cells through different molecular targets. Biochem. Pharmacol. 2020, 175, 113859. [CrossRef] [PubMed]

24. D'Aniello, E.; Iannotti, F.; Falkenberg, L.; Martella, A.; Gentile, A.; De Maio, F.; Ciavatta, M.; Gavagnin, M.; Waxman, J.; Di Marzo, V.; et al. In Silico Identification and Experimental Validation of (-)-Muqubilin A, a Marine Norterpene Peroxide, as PPAR $\alpha / \gamma-\operatorname{RXR} \alpha$ Agonist and RAR $\alpha$ Positive Allosteric Modulator. Mar. Drugs 2019, 17, 110. [CrossRef]

25. Vitale, R.; D’Aniello, E.; Gorbi, S.; Martella, A.; Silvestri, C.; Giuliani, M.; Fellous, T.; Gentile, A.; Carbone, M.; Cutignano, A.; et al. Fishing for Targets of Alien Metabolites: A Novel Peroxisome Proliferator-Activated Receptor (PPAR) Agonist from a Marine Pest. Mar. Drugs 2018, 16, 431. [CrossRef]

26. Carreras, J.; Kirillova, M.S.; Echavarren, A.M. Synthesis of (-)-Cannabimovone and Structural Reassignment of Anhydrocannabimovone through Gold(I)-Catalyzed Cycloisomerization. Angew. Chemie Int. Ed. 2016, 55, 7121-7125. [CrossRef]

27. Mueckler, M. Insulin resistance and the disruption of Glut4 trafficking in skeletal muscle. J. Clin. Invest. 2001, 107, 1211-1213. [CrossRef]

28. Martinez, L.; Berenguer, M.; Bruce, M.C.; Le Marchand-Brustel, Y.; Govers, R. Rosiglitazone increases cell surface GLUT4 levels in 3T3-L1 adipocytes through an enhancement of endosomal recycling. Biochem. Pharmacol. 2010, 79, 1300-1309. [CrossRef]

29. Nakamura, S.; Takamura, T.; Matsuzawa-Nagata, N.; Takayama, H.; Misu, H.; Noda, H.; Nabemoto, S.; Kurita, S.; Ota, T.; Ando, H.; et al. Palmitate Induces Insulin Resistance in H4IIEC3 Hepatocytes through Reactive Oxygen Species Produced by Mitochondria. J. Biol. Chem. 2009, 284, 14809-14818. [CrossRef]

30. Feng, X.-T.; Wang, T.-Z.; Leng, J.; Chen, Y.; Liu, J.-B.; Liu, Y.; Wang, W.-J. Palmitate Contributes to Insulin Resistance through Downregulation of the Src-Mediated Phosphorylation of Akt in C2C12 Myotubes. Biosci. Biotechnol. Biochem. 2012, 76, 1356-1361. [CrossRef] 
31. Mo, J.; Zhou, Y.; Yang, R.; Zhang, P.; He, B.; Yang, J.; Li, S.; Shen, Z.; Chen, P. Ginsenoside Rg1 ameliorates palmitic acid-induced insulin resistance in HepG2 cells in association with modulating Akt and JNK activity. Pharmacol. Rep. 2019, 71, 1160-1167. [CrossRef] [PubMed]

32. Acton, A.; Banck, M.; Bréfort, J.; Cruz, M.; Curtis, D.; Hassinen, T.; Heikkilä, V.; Hutchison, G.; Huuskonen, J.; Jensen, J.; et al. Chemical 2.00; Department of Chemistry, University of Kuopio: Kuopio, Finland, 2006.

33. Clark, M.; Cramer, R.D.; Van Opdenbosch, N. Validation of the general purpose tripos 5.2 force field. J. Comput. Chem. 1989, 10, 982-1012. [CrossRef]

34. Schmidt, M.W.; Baldridge, K.K.; Boatz, J.A.; Elbert, S.T.; Gordon, M.S.; Jensen, J.H.; Koseki, S.; Matsunaga, N.; Nguyen, K.A.; Su, S.; et al. General atomic and molecular electronic structure system. J. Comput. Chem. 1993, 14, 1347-1363. [CrossRef]

35. Fox, T.; Kollman, P.A. Application of the RESP Methodology in the Parametrization of Organic Solvents. J. Phys. Chem. B 1998, 102, 8070-8079. [CrossRef]

36. Morris, G.M.; Huey, R.; Lindstrom, W.; Sanner, M.F.; Belew, R.K.; Goodsell, D.S.; Olson, A.J. AutoDock4 and AutoDockTools4: Automated docking with selective receptor flexibility. J. Comput. Chem. 2009, 30, 2785-2791. [CrossRef] [PubMed]

37. Götz, A.W.; Williamson, M.J.; Xu, D.; Poole, D.; Le Grand, S.; Walker, R.C. Routine Microsecond Molecular Dynamics Simulations with AMBER on GPUs. 1. Generalized Born. J. Chem. Theory Comput. 2012, 8, 1542-1555. [CrossRef]

38. Wang, J.; Wolf, R.M.; Caldwell, J.W.; Kollman, P.A.; Case, D.A. Development and testing of a general amber force field. J. Comput. Chem. 2004, 25, 1157-1174. [CrossRef]

39. Ryckaert, J.-P.; Ciccotti, G.; Berendsen, H.J.C. Numerical integration of the cartesian equations of motion of a system with constraints: Molecular dynamics of n-alkanes. J. Comput. Phys. 1977, 23, 327-341. [CrossRef]

40. Pettersen, E.F.; Goddard, T.D.; Huang, C.C.; Couch, G.S.; Greenblatt, D.M.; Meng, E.C.; Ferrin, T.E. UCSF Chimera-A visualization system for exploratory research and analysis. J. Comput. Chem. 2004, 25, 1605-1612. [CrossRef]

41. Iannotti, F.A.; Panza, E.; Barrese, V.; Viggiano, D.; Soldovieri, M.V.; Taglialatela, M. Expression, Localization, and Pharmacological Role of $\mathrm{K}_{\mathrm{v}} 7$ Potassium Channels in Skeletal Muscle Proliferation, Differentiation, and Survival after Myotoxic Insults. J. Pharmacol. Exp. Ther. 2010, 332, 811-820. [CrossRef]

(C) 2020 by the authors. Licensee MDPI, Basel, Switzerland. This article is an open access article distributed under the terms and conditions of the Creative Commons Attribution (CC BY) license (http://creativecommons.org/licenses/by/4.0/). 